\title{
Prevention of MRSA pneumonia by oral vancomycin decontamination: a randomised trial
}

\author{
L. Silvestri*, H.K.F. van Saene* ${ }^{\#}$ M. Milanese*, F. Fontana ${ }^{\Uparrow}$, D. Gregori ${ }^{+}$, L. Oblach*, N. Piacente*, \\ M. Blazic*
}

Prevention of MRSA pneumonia by oral vancomycin decontamination: a randomised trial. L. Silvestri, H.K.F. van Saene, M. Milanese, F. Fontana, D. Gregori, L. Oblach, N. Piacente, M. Blazic. (C) ERS Journals Ltd 2004.

ABSTRACT: This study was undertaken to assess whether oropharyngeal vancomycin may control oropharyngeal carriage and lower airway infection due to methicillinresistant Staphylococcus aureus (MRSA) acquired in the intensive care unit (ICU). Secondary endpoints were the emergence of vancomycin-resistant enterococci, vancomycin-intermediate $S$. aureus and vancomycin consumption.

A total of 84 patients, admitted to a medical/surgical ICU and mechanically ventilated for $>72 \mathrm{~h}$, were randomly assigned to control $(n=42)$ or test $(n=42)$ group. Both groups received the protocol of selective decontamination of the digestive tract, including polymyxin E, tobramycin and amphotericin B. Patients in the test group received $0.5 \mathrm{~g}$ of a $4 \%$ vancomycin gel at 6 -h intervals in the oropharynx.

Lower airway infections due to MRSA acquired on the ICU were reduced in the test group, as was oropharyngeal carriage. Neither vancomycin-resistant enterococci nor vancomycin-intermediate $S$. aureus were isolated from either surveillance or diagnostic samples during the study period. The vancomycin costs were lower in the test group.

This study demonstrates that oropharyngeal vancomycin, which controls intensive care unit-acquired lower airway infections and secondary carriage due to methicillinresistant Staphylococcus aureus, is cost-effective and safe in terms of vancomycinresistant enterococci and vancomycin-intermediate Staphylococcus aureus.

Eur Respir J 2004; 23: 921-926.
*Dept of Emergency, Unit of Anaesthesia and Intensive Care, and Unit of Microbiology and Laboratory Medicine, Regional Hospital of Gorizia, Gorizia, and ${ }^{+}$Dept of Public Health and Microbiology, University of Turin, Turin, Italy. ${ }^{\#}$ Dept of Medical Microbiology, University of Liverpool, Liverpool, UK.

Correspondence: L. Silvestri, Unità Operativa di Anestesia e Rianimazione, Presidio Ospedaliero, Via Vittorio Veneto 171, 34170 Gorizia, Italy.

Fax: 390481592977

E-mail: lucianosilvestri@yahoo.it

Keywords: Carrier state

intensive care unit

methicillin resistance

pneumonia

Staphylococcus aureus

vancomycin

Received: September 302003

Accepted after revision: February 92004
Aspiration of contaminated oropharyngeal secretions is the main pathway for the development of lower airway infections in patients requiring mechanical ventilation on the intensive care unit (ICU) [1]. Individuals with an underlying disease are often oropharyngeal carriers of abnormal aerobic Gramnegative bacilli (AGNB) [2] and methicillin-resistant Staphylococcus aureus (MRSA) [3]. Prevention and eradication of the abnormal carrier state can be achieved by the administration of nonabsorbable antimicrobials into the oropharynx, as part of selective decontamination of the digestive tract (SDD) [4].

The European Task Force on ventilator-associated pneumonia [5] and the Consensus Conference in Critical Care on ICU-acquired pneumonia [6] acknowledged SDD as an evidence-based manoeuvre that reduces both pneumonia and mortality. These recommendations were based on two metaanalyses of randomised controlled trials [7, 8]. Recently, two individual trials of huge sample size confirmed the metaanalytic evidence $[9,10]$. The SDD strategy, using both parenteral and enteral antibiotics, significantly reduced pneumonia and other lower airway infections, combined with $8 \%$ absolute mortality reduction. The impact of SDD was similar in medical and surgical patients. Surveillance cultures are recommended to detect emergence of resistance.

Three SDD trials included oropharyngeal vancomycin in order to clear MRSA, which is intrinsically resistant to polymyxin and aminoglycosides, from the oropharyngeal cavity [11-13]. However, a separate analysis of the impact of topical vancomycin on MRSA lower airway infections was not performed in any of these studies.

A randomised trial was conducted to assess the impact of a $4 \%$ vancomycin gel, applied into the oropharynx, on MRSA lower airway infections in patients treated on a medical/ surgical ICU. Secondary endpoints were carriage rates and indices of MRSA in the oropharynx, the emergence of vancomycin-resistant enterococci (VRE), as well as vancomycinintermediate $S$. aureus (VISA) and vancomycin consumption.

\section{Material and methods}

\section{Study subjects}

This study was conducted over a 1-yr period, in an eightbedded ICU, at a multidisciplinary regional-referral hospital. The ICU is a facility with an annual admission rate of $\sim 350$ patients; medical patients account for $80 \%$ of the population, the remaining $20 \%$ are surgical and trauma patients. For isolation purposes, patients can be cohorted in to three, two-bedded cubicles and if necessary, in to a separate highdependency unit. Patients with multiresistant microorganisms were always isolated.

All consecutive patients aged $>18$ yrs, who were expected 
to require mechanical ventilation for $>72 \mathrm{~h}$, were enrolled. Exclusion criteria were pregnancy, known allergy to the study antibiotic, discharge, death or extubation during the initial $72 \mathrm{~h}$ of the protocol. Enteral feeding through a nasogastric or a naso-jejunum tube or a percutaneous gastrostomy was administered, with progression to full nutritional support within $96 \mathrm{~h}$. Stress gastric-ulcer prophylaxis with $\mathrm{H}_{2-}$ antagonists was not routinely practiced; sucralfate was not used. A semi-recumbent position of $30^{\circ}$ was maintained during mechanical ventilation. Subglottic suctioning was not practiced. Patients requiring ventilation of $\geqslant 7$ days received a percutaneous tracheostomy. The study ended with extubation, discharge or death of the patient. The study was approved by the local ethical committee. Informed consent was obtained before randomisation from either the patient or the patient's next of kin, according to competency.

Infection prophylaxis, using the original protocol of SDD, was routinely applied in all patients. The SDD protocol consisted of a combination of enteral (i.e. polymyxin $\mathrm{E}$, tobramycin and amphotericin B) and parenteral antimicrobials, hygiene and surveillance cultures $[14,15]$. A gel $(0.5 \mathrm{~g})$ containing $2 \%$ polymyxin $\mathrm{E}$, tobramycin, and amphotericin $\mathrm{B}$ was applied in the oropharynx four times $\cdot$ day $^{-1}$, and $10 \mathrm{~mL}$ of a suspension containing $100 \mathrm{mg}$ polymyxin E, $80 \mathrm{mg}$ tobramycin and $500 \mathrm{mg}$ amphotericin B was administered four times day $^{-1}$ through the nasogastric tube. A 4-day course of systemic cefotaxime (or ceftazidime if the patient was suspected of carrying a pseudomonal strain) was part of the SDD protocol. The standards of hygiene recommended by the Centers for Disease Control and Prevention (CDC) were followed. Surveillance samples of throat and rectum were taken on admission and then twice weekly (Monday and Thursday).

Systemic antibiotics, including vancomycin, were administered in case of infection, based on clinical signs and laboratory results. In general, a 5-day course of antibiotics was given, followed by clinical re-evaluation of the patient.

\section{Study design}

After obtaining the informed consent, patients were randomly allocated to either control or test group by a computer-generated random-number table, which was not available on the ICU. The doctor in charge of the enrolled patient contacted the investigator (D. Gregori, Dept of Public Health and Microbiology, University of Turin, Turin, Italy), who was not involved in patient care for the randomisation. Patients who were assigned to the test group received $0.5 \mathrm{~g}$ of a $4 \%$ vancomycin gel at 6-h intervals, applied in the oropharynx with a gloved finger. The radiologist and the microbiologist were blinded to the patient groups. Results of surveillance cultures were withheld by the microbiologist. Care providers were not blinded to the study and patients' allocation, except two physicians (L. Silvestri, Dept of Emergency, Unit of Anaesthesia and Intensive Care, Regional Hospital of Gorizia, Gorizia, Italy and H.K.F. van Saene, Dept of Medical Microbiology, University of Liverpool, Liverpool, UK) who participated in the endpoint review committee.

Age, sex, admission diagnosis, and simplified acute physiology score (SAPS) II were obtained on admission. Patients with lower airway infection, number of infection episodes, MRSA carriage rate and index, classification of infection episodes according to the carrier state, length of ICU stay, days of ventilation, day of onset of infection and carriage, and vancomycin consumption were recorded.

\section{Methods}

Surveillance samples of throat and gut were processed qualitatively and semi-quantitatively to detect the level of carriage. Throat and rectal swabs were cultured minimally on to three solid media, including a staphylococcal, yeast and MacConkey agar plate. Each swab was streaked onto the solid media using the three-segment method [16, 17]. Standard methods for identification, typing, and sensitivity patterns were used for all microorganisms. To differentiate $S$. aureus from other species of staphylococci, the laboratory used production of DNAse (by a DNA agar plate method), a slideagglutination test to detect clumping factor and protein A (Prolex $^{\text {TM }}$ Staph Latex Kit; Prolab Diagnostics, Neston, Wirral, UK), and a slide agglutination kit for the rapid detection of penicillin-binding protein-2a for MRSA (MRSA Screen; Biogenetics, Padova, Italy). If the results were inconclusive, staphylococcal species were identified by a biochemical method (ID 32 Staph; BioMerieux, Marcy l'Etoile, France). Sensitivity patterns were determined by the ATB and Vitek system (Gram-positive susceptibility (GPS)-517 for staphylococci, GPS-516 for enterococci, Gram-negative susceptibility-502 for Gram-negative rods; BioMerieux). MRSA isolates which had a minimum inhibitory concentration of $>256 \mu \mathrm{g} \cdot \mathrm{mL}^{-1}$ on gradient plates in the exponential antibiotic gradient (E) test were confirmed to be MRSA. The breakpoints for VRE and VISA were $16 \mu \mathrm{g} \cdot \mathrm{mL}^{-1}$ and $8 \mu \mathrm{g} \cdot \mathrm{mL}^{-1}$ of vancomycin, respectively $[18,19]$.

Diagnostic samples, including tracheal aspirate, urine and blood, were obtained only on clinical indication and processed according to the method described previously. Identification, typing and extended sensitivity patterns were used to confirm or refute the relatedness of microorganisms.

Carriage or carrier state was defined as the patient's state when the same strain of potentially pathogenic microorganism (PPM) was isolated from at least two consecutive surveillance samples, in any concentration, over a period of 1 week [20]. Carriage index was defined as the sum of growth densities of the positive surveillance cultures divided by the total number of swabs [17].

Lower respiratory tract infection (LRTI) included pneumonia and tracheobronchitis. Pneumonia was diagnosed upon the presence of a new and/or progressive pulmonary infiltrate on chest radiograph persistent for $>48 \mathrm{~h}$, in addition to two clinical criteria and one confirmation criterion. Clinical criteria included the following: 1) core temperature $\geqslant 38.3^{\circ} \mathrm{C}$ or $<36^{\circ} \mathrm{C}$; 2) leucocytosis $>12 \times 10^{9} \cdot \mathrm{L}^{-1}$ or leucopenia $<4 \times 10^{9} \cdot \mathrm{L}^{-1}$; and 3) purulent tracheobronchial secretions. Confirmation criteria included one of the following: 1) endotracheal aspirate with semiquantitative cultures at a concentration of $\geqslant 10^{5} ; 2$ ) positive blood culture in the absence of an extrapulmonary focus; and 3) a response to antimicrobial therapy in the absence of alternative diagnosis [21]. Tracheobronchitis was defined using the same criteria as pneumonia, omitting the radiographical findings.

Infections were classified according to the criterion of the carrier state [20]. Primary endogenous infections were caused by both community and hospital PPMs carried by the patient in throat and/or gut on admission to the ICU. Secondary endogenous infections were caused by PPMs not carried in throat and/or gut at the time of admission to the ICU, but acquired in the throat and/or gut during an ICU stay, prior to the infection. Surveillance and diagnostic samples yielded the same microorganism in infections of endogenous origin. Exogenous infections were caused by PPMs introduced into the patients from the environment, either animate or inanimate. Bacteria were transferred directly into an internal organ, without previous carriage. In exogenous infections, 
surveillance samples were negative for PPMs that readily appeared in diagnostic samples. Only secondary endogenous and exogenous infections were labelled ICU-acquired infections; whilst primary endogenous infections were considered to be imported infections.

Defined daily doses (DDD) of vancomycin were $2 \mathrm{~g}$ and $80 \mathrm{mg}$ of parenteral and oral vancomycin, respectively [22].

\section{Analysis}

The study was designed to test the hypothesis that oropharyngeal vancomycin would reduce the incidence of lower airway infection due to MRSA. The current authors have previously determined a baseline incidence of MRSA ICU-acquired lower airway infection of $35 \%$. A sample size of 84 patients was calculated to be necessary to provide a reduction in the incidence of MRSA lower airway infection from $35 \%$ to $10 \%$, with a statistical power of 0.80 by a twosided test at the 5\% significance level.

Data are presented as median (interquartile range), or percentages, as appropriate. Exploratory data analyses were performed, analysing categorical variables with Chi-squared test or Fischer's exact test when appropriate. Comparisons between continuous variables among two groups were carried out with unpaired t-test for variables with normal distribution, and Mann-Whitney U-test for non-normally distributed variables. Effects were evaluated using a modelling strategy. Treatment effect was adjusted for age, sex and SAPS II score as covariates. The regression model was chosen according to the outcome characteristics: logit (probability of experiencing an infection), log-linear (number of infections and number of days of carriage) and multinomial-logit (probability of experiencing a specific type of infection) models were used for modelling the outcome. The proportional hazard model was used for estimating the change in infection rates with respect to time [23]. Confidence intervals $(95 \% \mathrm{CI})$ were computed for days when curves differentiated.

\section{Results}

A total of 84 patients completed the trial: 42 patients in each group. Table 1 shows the baseline characteristics of the

Table 1.-Baseline characteristics of the patients studied

\begin{tabular}{lcc}
\hline Characteristic & Control & Vancomycin \\
\hline Subjects n & 42 & 42 \\
Age yrs & $69.5(63-76)$ & $72(64.7-77)$ \\
Sex male & $24(57)$ & $29(69)$ \\
SAPS II score & $45.5(34.5-55.2)$ & $43.5(35.2-56)$ \\
Admission diagnosis & & \\
$\quad$ Cardiac & $5(12)$ & $8(19)$ \\
COPD & $10(24)$ & $16(38)$ \\
Pneumonia at entry & $7(17)$ & $10(24)$ \\
Major surgery & $2(5)$ & $2(5)$ \\
Gastrointestinal disease & $3(7)$ & $4(10)$ \\
Neurological diseases & $17(40)$ & $16(38)$ \\
Trauma & $5(12)$ & $2(5)$ \\
Sepsis & $3(7)$ & $2(5)$ \\
Renal failure & $2(5)$ & $7(17)$ \\
Diabetes & $6(14)$ & $17(40)$ \\
Receiving antibiotics at study entry & $13(31)$ &
\end{tabular}

Data are presented as median (interquartile range) and $\mathrm{n}(\%)$, unless otherwise stated. More than one admission diagnosis condition per patient is possible. SAPS: simplified acute physiology score; COPD: chronic obstructive pulmonary disease.
Table 2. - Intensive care unit characteristics of the population studied

\begin{tabular}{|c|c|c|}
\hline Characteristic & Control & Vancomycin \\
\hline Subjects n & 42 & 42 \\
\hline Length of stay days & $15.5(8.7-34.5)$ & $13(9.7-24)$ \\
\hline \multicolumn{3}{|l|}{ Days of ventilation } \\
\hline Total n & 649 & 621 \\
\hline Median (interquartile range) & $10(6-22.2) \quad 1$ & $11.5(7.7-18.5)$ \\
\hline \multicolumn{3}{|l|}{ Tracheotomy } \\
\hline Number of patients & $15(36)$ & $17(40)$ \\
\hline Day of placement & $14(9-21)$ & $10(2.5-14.5)$ \\
\hline \multicolumn{3}{|c|}{$\mathrm{H}_{2}$-receptor blocking drugs therapy } \\
\hline Number of patients & $19(45)$ & $18(43)$ \\
\hline Days of therapy & $0(0-5.25)$ & $0(0-4.25)$ \\
\hline \multicolumn{3}{|l|}{ Patients receiving nutrition } \\
\hline Parenteral & $11(26)$ & $9(21)$ \\
\hline Enteral & $34(81)$ & $38(90)$ \\
\hline Nasogastric tube & $42(100)$ & $42(100)$ \\
\hline Jejunal tube & $2(5)$ & $1(2)$ \\
\hline Gastrostomy & $3(7)$ & $2(5)$ \\
\hline Patients with infection & $16(38)$ & $24(57)$ \\
\hline $\begin{array}{l}\text { Total of infection episodes } n \\
\text { Type of infection }\end{array}$ & Type of infection & 31 \\
\hline Tracheobronchitis & $2(7)$ & $2(7)$ \\
\hline Pneumonia & $19(63)$ & $22(71)$ \\
\hline Urinary tract & $3(10)$ & \\
\hline Bloodstream & $4(13)$ & $3(10)$ \\
\hline Other & $2(7)$ & $4(13)$ \\
\hline \multicolumn{3}{|c|}{ Classification of infection episodes } \\
\hline Primary endogenous & $15(50)$ & $22(71)$ \\
\hline Secondary endogenous & $10(33)$ & $3(10)$ \\
\hline Exogenous & $5(17)$ & $6(19)$ \\
\hline Day of infection onset & $1(1-13)$ & $1(1-10)$ \\
\hline Mortality & $15(36)$ & $12(29)$ \\
\hline
\end{tabular}

Data are presented as median (interquartile range) and $\mathrm{n}(\%)$, unless otherwise stated. For patients receiving nutrition, more than one condition per patient is possible.

control and test groups. They were comparable between the two groups; the higher number of patients with chronic obstructive pulmonary disease in the vancomycin group was not significant, as compared to the control group. Table 2 shows the ICU characteristics. There were no significant differences between the two groups; although, the test group comprised of a higher number of infected patients, mainly due to the higher number of primary endogenous infections and less secondary endogenous infections.

A causative pathogen was identified in 46 out of 61 infection episodes. A total of 15 infections (six in the control and nine in the test group) were not microbiologically proven. A detailed list of pathogens is shown in table 3 .

Six patients receiving vancomycin developed six episodes of LRTI due to MRSA: five primary endogenous and one exogenous (table 4). Eight control patients had 10 lower airway infections due to MRSA: three primary endogenous, one exogenous and six secondary endogenous. The $4 \%$ vancomycin gel effectively prevented secondary endogenous lower airway infections in the test group ( 0 versus $6, \mathrm{p}<0.001)$. As only secondary endogenous and exogenous infections are nosocomial (i.e. ICU acquired), there were significantly less ICU-acquired lower airway infections in the test, as compared to the control group (1 versus 7 ; odds ratio (OR): $0.26 ; 95 \%$ CI: 0.08-0.88; $\mathrm{p}<0.001)$. Placement of a tracheostomy did not influence the lower airway infection rate, as only one exogenous infection developed after the tracheostomy in the control group. Patients in the test group stayed in the ICU significantly shorter compared to the control group (21.5 days 
Table 3.-Microorganisms causing the 61 episodes of infection in the patients studied

\begin{tabular}{|c|c|c|}
\hline Microorganisms & Control & Vancomycin \\
\hline \multicolumn{3}{|l|}{ Normal flora } \\
\hline MSSA & $1(3.3)$ & $1(3.2)$ \\
\hline Streptococcus pneumoniae & & $1(3.2)$ \\
\hline Haemophilus influenzae & $1(3.3)$ & \\
\hline Moraxella catarrhalis & $1(3.3)$ & \\
\hline CNS & $1(3.3)$ & $1(3.2)$ \\
\hline Yeasts & & $2(6.4)$ \\
\hline Anaerobes & $1(3.3)$ & \\
\hline \multicolumn{3}{|l|}{ Abnormal flora } \\
\hline Pseudomonas spp. & $5(16.7)$ & $7(22.6)$ \\
\hline Klebsiella spp. & & $1(3.2)$ \\
\hline Citrobacter spp. & $1(3.3)$ & $1(3.2)$ \\
\hline Morganella spp. & $1(3.3)$ & \\
\hline MRSA & $12(40)$ & $8(25.8)$ \\
\hline No growth & $6(20)$ & $9(29)$ \\
\hline
\end{tabular}

Data are presented as n (\%). MSSA: methicillin-sensitive Staphylococcus aureus; CNS: coagulase-negative staphylococci; MRSA: methicillinresistant Staphylococcus aureus.

versus 39 days, $\mathrm{p}<0.05)$. In the study population, seven patients (6.88-7.12) needed to receive the vancomycin gel to prevent one ICU-acquired MRSA lower airway infection.

A total of 28 patients carried MRSA in the oropharynx during the study period: 20 in the control group and eight in the test group $(\mathrm{p}<0.05$; table 4$)$. There were five oropharyngeal MRSA carriers on admission in the control group; whilst eight patients of the test group imported MRSA in their oral admission flora. The 4\% vancomycin gel completely prevented acquisition and secondary MRSA carriage (OR: 0.25 ; $95 \%$ CI: $0.09-0.69$; $\mathrm{p}=0.007)$. In the study population, 2.8 patients $(2.65-2.94)$ needed to be treated with vancomycin to prevent one oropharyngeal carrier of MRSA. The median oropharyngeal MRSA carriage index was significantly higher in the control than in the test group ( 1.78 versus $0.05 ; \mathrm{p}<0.01)$. Moreover, a conservative estimate adjusted for age, sex and SAPS II, indicated that the duration of oropharyngeal carriage significantly decreased from 508 days $\cdot 1,000$ ventilator days ${ }^{-1}$ in the control group to 76 days $\cdot 1,000$ ventilator days $^{-1}$ in the treatment group $(\mathrm{p}<0.01)$.

In order to treat 12 episodes of MRSA infection in the control group, 93.5 DDD of vancomycin were dispensed by the pharmacy (mean: $16 \mathrm{~g}$; median length of therapy: 9 days).
In contrast, in the test group, 42.5 DDD of vancomycin were used to treat eight episodes (mean: $11 \mathrm{~g}$; median length of therapy: 6.5 days). A total of $52 \mathrm{~g}(650 \mathrm{DDD})$ of topical vancomycin, given in 2,627 gel applications, were administered to the test patients. The cost of both enteral and parenteral vancomycin was $€ 3,120$ in the control group and $€ 2,294$ in the test group.

A total of 979 microbiological samples were processed: 406 surveillance and 70 diagnostic samples in the control group, and 418 surveillance and 85 diagnostic samples in the test group. Neither VISA nor VRE were isolated during the study period. During the study period and the following year, neither VRE nor VISA were found throughout the hospital.

\section{Discussion}

This 1-yr randomised trial, in 84 patients requiring longterm ventilation, shows that oropharyngeal decontamination, using 4\% vancomycin gel, prevents nosocomial lower airway infections due to MRSA ( 7 versus 1 ) and reduces both carrier rates $(47 \%$ versus $19 \%)$ and median carriage indices $(1.78$ versus 0.05 ). Less vancomycin, both topical and systemic, was used in the test group as compared to the control group that received solely i.v. vancomycin. Finally, neither VRE nor VISA were detected in any of the diagnostic and surveillance samples.

First, the manoeuvre of $4 \%$ oropharyngeal vancomycin impacts only infections of the lower airways due to MRSA acquired on the unit [24]. Patients with a lower airway infection (incubating or established on admission) were unlikely to benefit from the $4 \%$ gel. Secondly, infections other than lower airway infections, including septicaemia and wound infections, were not expected to be impacted by the study manoeuvre [11-13, 25]. Thirdly, exogenous lower airway infections due to MRSA, in particular in tracheotomised patients, cannot be controlled, as they are not preceded by carriage in the oropharynx [26]. A substantial number of critically ill patients were referred from other hospitals in the region to the ICU. Of the 84 patients, $13(15 \%)$ were oropharyngeal carriers of MRSA on arrival to the ICU and eight subsequently developed a primary endogenous lower airway infection: three in the control and five in the test group. Of the 20 MRSA infection episodes, 16 were lower airway infections. A third of the patients (32 out of 84 ) received a tracheostomy at a median of 14 and 10 days in the

Table 4.-Methicillin-resistant Staphylococcus aureus lower airway infection and oropharyngeal carriage

\begin{tabular}{|c|c|c|c|}
\hline & Control & Vancomycin & p-value \\
\hline Subjects n & 42 & 42 & \\
\hline \multicolumn{4}{|l|}{ Infection } \\
\hline Patients & $8(19)$ & $6(14)$ & NS \\
\hline Number of episodes & 10 & 6 & NS \\
\hline Primary endogenous & $3(30)$ & $5(83)$ & NS \\
\hline Secondary endogenous & $6(60)$ & & $<0.01$ \\
\hline Exogenous & $1(10)$ & $1(17)$ & NS \\
\hline Day of onset & $10(6.75-22)$ & $1(1-8)$ & $<0.01$ \\
\hline Nosocomial day of onset & $12(7-21)$ & 6 & $<0.05$ \\
\hline Length of stay days & $39(36-53)$ & $21.5(16-28.5)$ & $<0.05$ \\
\hline \multicolumn{4}{|l|}{ Carriage } \\
\hline Patients & $20(47)$ & $8(19)$ & $<0.05$ \\
\hline On admission & $5(12)$ & $8(19)$ & NS \\
\hline ICU-acquired & $15(36)$ & & $<0.01$ \\
\hline Duration of carriage days & 508 & 76 & $<0.01$ \\
\hline Index & $1.78(0.91-2.24)$ & $0.05(0.007-0.17)$ & $<0.01$ \\
\hline
\end{tabular}

Data are presented as n, $\mathrm{n}(\%)$ and median (interquartile range), unless otherwise stated. ICU: intensive care unit. 
control and test group, respectively. There was one patient with an exogenous infection in each group. The remaining six infections due to MRSA all occurred in the control group, as the $4 \%$ vancomycin gel effectively prevented MRSA acquisition and subsequent secondary carriage. It should be appreciated that this analysis is only possible with knowledge of the carrier state provided by surveillance samples [16, 24]. Regular monitoring of the MRSA carrier state, on admission and twice weekly, allows the distinction of primary endogenous infections, due to imported MRSA from exogenous and secondary endogenous infections, caused by MRSA acquired on the ICU, i.e. "true" nosocomial infections.

All patients in the study received the full SDD protocol, including topical polymyxin E/tobramycin/amphotericin $\mathrm{B}$, as prophylaxis against methicillin-sensitive $S$. aureus, AGNB and fungal infections $[4,15]$. However, by design, the classical protocol of SDD does not cover MRSA. There are three prospective, randomised, double-blind, placebo-controlled studies of SDD which included oropharyngeal vancomycin in their protocol [11-13]. Those studies were not specifically designed to evaluate the impact of vancomycin on MRSA pneumonia. PUGIN et al. [11] showed a reduction in $S$. aureus oropharyngeal carriage and pneumonia due to Gram-positive cocci, in patients treated with $6 \times 250 \mathrm{mg} \cdot \mathrm{day}^{-1}$ of vancomycin solution instilled into the retropharynx. In a study by KORINEK et al. [12], which included a 4\% vancomycin paste, $S$. aureus was the main pathogen responsible for $60 \%$ of all lower airway infections. However, no significant differences in tracheal colonisation and pneumonia due to $S$. aureus were shown. In a recent study by Bergmans et al. [13], using 2\% vancomycin applied in the oropharynx, no data on MRSA carriage and pneumonia were reported. In these three studies, among $S$. aureus isolates, MRSA was not mentioned [11], or was not isolated $[12,13]$.

In the present study, a vancomycin gel was used, rather than a solution, in order to guarantee a prolonged contact time between the MRSA carried in the oropharynx and the topical vancomycin, and a high bactericidal concentration. The intervention significantly reduced rates of carriers and indices of carriage. More specifically, there was no acquisition of MRSA in the test group, whilst 15 control patients acquired MRSA and became oral carriers during the study. The patients receiving $4 \%$ vancomycin had a median index of carriage of 0.05 , i.e. $<10^{3} \mathrm{MRSA} \cdot \mathrm{mL}^{-1}$ of saliva. Overgrowth, defined as $\geqslant 10^{5} \cdot \mathrm{mL}^{-1}$ of saliva, has been shown to promote colonisation and infection of lower airways [1]. The significantly reduced MRSA concentrations in the oropharynx may explain the absence of secondary endogenous lower airway infections.

A recent Canadian trial demonstrated that ventilated patients who developed pneumonia stayed in ICU for 4.3 days longer than matched controls [27]. This is in line with the current authors' observations that patients with lower airway infections due to MRSA spent more days on the unit (38 and 21 days in the control and test group, respectively). The substantial impact of oropharyngeal vancomycin on ICUacquired lower airway infections in this trial was associated with lower vancomycin costs.

VRE [28] and VISA [19] are present in many countries, and the Hospital Infection Control Practices Advisory Committee, in collaboration with the $\mathrm{CDC}$, has issued recommendations for their control $[29,30]$. One of these recommendations is that oral vancomycin should not be used. In the current study, 42 patients received $80 \mathrm{mg}$ of topical vancomycin daily for a median of 11.5 days, its use was limited to minimise the risk of resistant strains emerging [13]. Surveillance and diagnostic cultures were screened for VRE and VISA, and were negative over the study period and during the following year. Overgrowth of enterococci and/or MRSA in the presence of nonlethal faecal vancomycin levels is hypothesised to promote the selection of resistant mutants amongst the enterococcal and staphylococcal population [31]. Topical oropharyngeal application of $80 \mathrm{mg}$ of vancomycin daily is unlikely to result in microbiologically active vancomycin levels in the faeces. Vancomycin is inactivated by faecal material, necrotic cells and fibres along its passage through the alimentary canal. The absence of VRE and VISA is in line with a recent situation during which intestinal vancomycin was administered to control an MRSA outbreak [32].

The current authors acknowledge important limitations of the study. First, the study was not designed as placebocontrolled, and care providers were not blinded. However, the radiologist and the microbiologist, and two ICU physicians who made the assessment, were blinded. Secondly, imbalances of underlying conditions on admission are an inherent problem in studies of small sample size and may potentially affect the results. Thirdly, the MRSA ICU-acquired lower airway infection rate in the control group was less than expected $(23 \%$ instead of $35 \%)$, possibly due to a contamination bias [33]. The study manoeuvre may have impacted the control group, as the likelihood of acquiring MRSA due to transmission was reduced. Finally, although the difference in rates of MRSA ICU-acquired lower airway infections between the two groups is significant, the number of events on which to base a solid inference is small.

In conclusion, the present study showed that topical oropharyngeal vancomycin was effective in preventing intensive care unit-acquired lower airway infections and secondary carriage due to methicillin-resistant Staphylococcus aureus in long-term mechanically ventilated patients receiving selective decontamination of the digestive tract. The manoeuvre was found to be cost-effective and safe in terms of isolation of vancomycin-resistant enterococci and vancomycin-intermediate Staphylococcus aureus. In an endemic methicillin-resistant Staphylococcus aureus setting, prophylactic decontamination with vancomycin may control nosocomial methicillin-resistant Staphylococcus aureus infections, particularly in units to which methicillin-resistant Staphylococcus aureus patient carriers are regularly referred. Surveillance cultures are mandatory to evaluate the effectiveness and safety of the prophylactic regimen. Further studies are required to confirm the current authors' findings, before the long-term safety of this approach can be determined.

\footnotetext{
Acknowledgements. The authors thank the nursing staff of the intensive care unit for their assistance in conducting the trial, and the personnel of the Microbiology for processing surveillance and diagnostic samples. The authors are indebted to G. Campestrini and the staff of the hospital pharmacy for their technical support in preparing the decontaminating drugs.
}

\section{References}

1. van Uffelen R, van Saene HKF, Fidler V, Lowenberg A. Oropharyngeal flora as a source of colonizing the lower airways in patients on artificial ventilation. Intensive Care Med 1984; 10: 233-237.

2. Mobbs KJ, van Saene HKF, Sunderland D, Davies PDO. Oropharyngeal bacillary carriage in chronic obstructive pulmonary disease: relation to severity of disease. Respir Med 1999; 93: 540-545.

3. Cox RA, Conquest C, Mallaghan C, Marples RR. A major outbreak of methicillin-resistant Staphylococcus aureus 
caused by new phage-type (EMRSA-16). J Hosp Infect 1995; 29: $87-106$

4. Stoutenbeek CP, van Saene HKF, Miranda DR, Zandstra DF, Langrehr D. The effect of oropharyngeal decontamination using topical nonabsorbable antibiotics on the incidence of nosocomial respiratory tract infections in multiple trauma patients. J Trauma 1987; 27: 357-364.

5. Torres A, Carlet J. Ventilator-associated pneumonia. European Task Force on ventilator-associated pneumonia. Eur Respir J 2001; 17: 1034-1045.

6. Hubmayr RD, Burchardi H, Elliot M, et al. Statement of the 4th International Consensus Conference in Critical Care on ICU-acquired pneumonia- Chicago, Illinois, May 2002. Intensive Care Med 2002; 28: 1521-1536.

7. D'Amico R, Pifferi S, Leonetti C, Torri V, Tinazzi A, Liberati A. Effectiveness of antibiotic prophylaxis in critically ill adult patients: systematic review of randomised controlled trials. Br Med J 1998; 316: 1275-1285.

8. Nathens AB, Marshall JC. Selective decontamination of the digestive tract in surgical patients. A systematic review of the evidence. Arch Surg 1999; 134: 170-176.

9. Krueger WA, Lenhart F-P, Neeser G, et al. Influence of combined intravenous and topical antibiotic prophylaxis on the incidence of infections, organ dysfunctions, and mortality in critically ill surgical patients. A prospective, stratified, randomized, double-blind, placebo-controlled clinical trial. Am J Respir Crit Care Med 2002; 166: 1029-1037.

10. de Jonge E, Schultz M, Spanjaard L, et al. Effects of selective decontamination of the digestive tract on mortality and acquisition of resistant bacteria in intensive care: a randomised controlled trial. Lancet 2003; 363: 1011-1016.

11. Pugin J, Auckenthaler R, Lew DP, Suter PM. Oropharyngeal decontamination decreases incidence of ventilatorassociated pneumonia. A randomized, placebo-controlled, double-blind clinical trial. JAMA 1991; 265: 2704-2710.

12. Korinek AM, Laisne MJ, Nicolas MH, Raskine L, Deroin V, Sanson-Lepors MJ. Selective decontamination of the digestive tract in neurosurgical intensive care units patientsa double-blind, randomized, placebo-controlled study. Crit Care Med 1993; 21: 1466-1473.

13. Bergmans DCJJ, Bonten MJM, Gaillard CA, et al. Prevention of ventilator-associated pneumonia by oral decontamination: a prospective, randomized, double-blind, placebo-controlled study. Am J Respir Crit Care Med 2001; 164: 382-388.

14. Silvestri L, Lenhart FP, Fox MA. Prevention of intensive care unit infections. Curr Anaesth Crit Care 2001; 12: 34-40.

15. van Saene HKF, Petros A, Ramsay G, Baxby D. All great truths are iconoclastic: selective decontamination of the digestive tract moves from heresy to level 1 truth. Intensive Care Med 2003; 29: 677-690.

16. Silvestri L, Monti-Bragadin C, Milanese M, et al. Are most ICU infections really nosocomial? A prospective observational cohort study in mechanically ventilated patients. J Hosp Infect 1999; 42: 125-133.

17. Damjanovic V, Connolly CM, van Saene HKF, et al. Selective decontamination with nystatin for control of a Candida outbreak in a neonatal intensive care unit. $J$ Hosp Infect 1993; 24: 245-259.

18. Morris JG, Shay DK, Hebden JN, et al. Enterococci resistant to multiple antimicrobial agents including vancomycin. Establishment of endemicity in a university medical center. Ann Intern Med 1995; 123: 250-259.

19. Guerin F, Buu-Hoi A, Mainardi JL, et al. Outbreak of methicillin-resistant Staphylococcus aureus with reduced susceptibility to glycopeptides in a Parisian hospital. J Clin Microbiol 2000; 38: 2985-2988.

20. Sarginson RE, Taylor N, van Saene HKF. Glossary of terms and definitions. Curr Anaesth Crit Care 2001; 12: 2-5.

21. Ewig S, Torres A, El-Elbiary M, et al. Bacterial colonization patterns in mechanically ventilated patients with traumatic and medical injury. Incidence, risk factors, and association with ventilator-associated pneumonia. Am J Respir Crit Care Med 1999; 159: 188-198.

22. National Nosocomial Infections Surveillance (NNIS) System. Intensive care antimicrobial resistance epidemiology (ICARE) surveillance report, data summary from January 1996 through December 1997. Am J Infect Control 1999; 27: 279-284.

23. Heller G. The Cox proportional hazard model with a partly linear relative risk function. Lifetime Data Anal 2001; 7: 255257.

24. de la Cal MA, Cerdà E, Garcia-Hierro P, et al. Pneumonia in severe burns: a classification according to the concept of the carrier state. Chest 2000; 119: 1160-1165.

25. Bonten MJM, Gaillard CA, Johanson WG, et al. Colonization in patients receiving and not receiving topical antimicrobial prophylaxis. Am J Respir Crit Care Med 1994; 150: $1332-1340$

26. Morar P, Makura Z, Jones A, et al. Topical antibiotics on tracheostoma prevents exogenous colonization and infection of lower airways in children. Chest 2000; 117: 513-518.

27. Heyland DK, Cook DJ, Griffith L, Keenan SP, BrunBuisson C. The attributable morbidity and mortality of ventilator-associated pneumonia in the critically ill patient. Am J Respir Crit Care Med 1999; 159: 1249-1256.

28. Hendrix CW, Hammond JMJ, Swoboda SM, et al. Surveillance strategies and impact of vancomycin-resistant enterococcal colonization and infection in critically ill patients. Ann Surg 2001; 233: 259-265.

29. Hospital Infection Control Practices Advisory Committee (HICPAC). Recommendations for preventing the spread of vancomycin resistance. Infect Control Hosp Epidemiol 1995; 16: $105-113$

30. Centers for Disease Control. Interim guidelines for prevention and control of staphylococcal infection associated with reduced susceptibility to vancomycin. Morb Mort Wkly Rep 1997; 46: 626-635.

31. Donskey CJ, Chowdhry TK, Hecker MT, et al. Effect of antibiotic therapy on the density of vancomycin-resistant enterococci in the stool of colonized patients. $N$ Engl J Med 2000; 343: 1925-1932.

32. Silvestri L, Milanese M, Oblach L, et al. Enteral vancomycin to control methicillin-resistant Staphylococcus aureus outbreak in mechanically ventilated patients. Am $J$ Infect Control 2002; 30: 391-399.

33. Feinstein AR. The role of the maneuver in cause-effect research. In: Feinstein AR, ed. Clinical Epidemiology. Philadelphia, WB Saunders, 1985; pp. 191-214. 